\title{
Computational Aspects of Cooperative Game Theory
}

\author{
Michael Wooldridge \\ Department of Computer Science, University of Liverpool, Liverpool L69 3BX, UK \\ mjw@liv.ac.uk
}

\begin{abstract}
The theory of cooperative games provides a rich mathematical framework with which to understand the interactions between self-interested agents in settings where they can benefit from cooperation, and where binding agreements between agents can be made. Our aim in this talk is to describe the issues that arise when we consider cooperative game theory through a computational lens. We begin by introducing basic concepts from cooperative game theory, and in particular the key solution concepts: the core and the Shapley value. We then introduce the key issues that arise if one is to consider the cooperative games in a computational setting: in particular, the issue of representing games, and the computational complexity of cooperative solution concepts.
\end{abstract}

